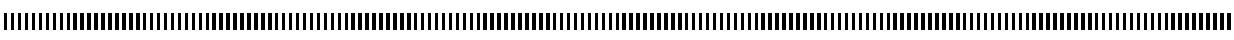

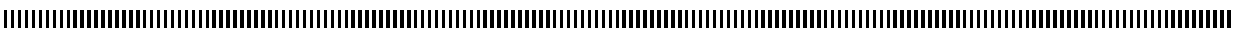

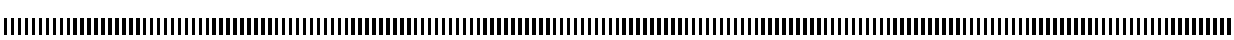

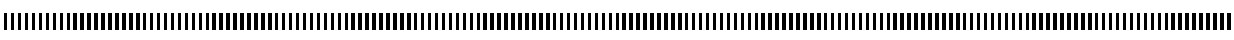

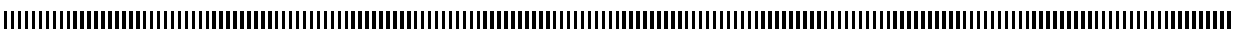

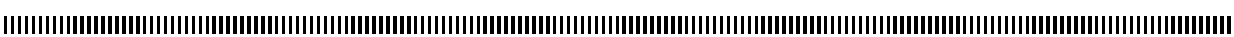

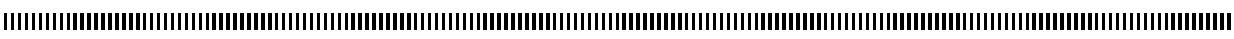

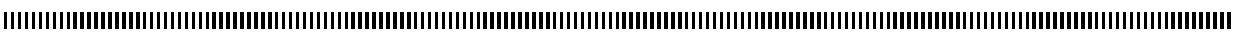

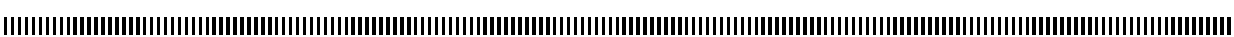

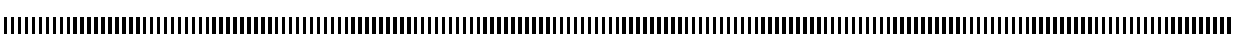

TAMTAM'09

\title{
Optimisation multicritère
}

\section{Une approche par partage des variables}

\author{
R. Aboulaich* - A. Habbal** - N. Moussaid* \\ * LERMA \\ Ecole Mohammadia d'Ingénieurs, \\ Avenue Ibn Sina B.P 765, Agdal, Rabat. Maroc. \\ aboulaich@emi.ac.ma,moussaid@unice.fr \\ ** LMJAD \\ Université de Nice Sophia-Antipolis, \\ Parc Valrose, 06108 Nice Cedex 2, France. \\ habbal@unice.fr
}

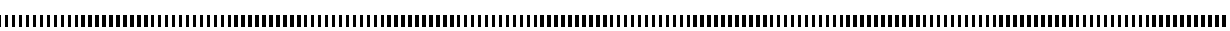

RÉSUMÉ. On s'intéresse, dans ce travail, à un problème d'optimisation multi-critère en utilisant la théorie des jeux. Ce problème est traité en utilisant de nouveaux algorithmes pour le partage de territoire dans le cas d'une optimisation concourante. Il s'agit de présenter une formulation de jeux de Nash entre deux joueurs en utilisant deux tableaux d'allocation. Chaque joueur minimise sa fonction coût en agissant sur les variables allouées par son propre tableau. Les deux tableaux sont à construire grâce à un algorithme itératif. Une application de ces algorithmes à un problème de traitement d'images est considérée.

ABSTRACT. We are interested here, in multi-criteria optimization problem using game theory. This problem will be treated by using a new algorithm for the splitting of territory in case of concurrent optimization, which presents a new formulation of Nash games between two players using two tables of allocations. Each player minimizes his cost function using the variables allocated by his own table. The two tables are given by an iterative algorithm. An image processing problem is addressed by using the proposed algorithms.

MOTS-CLÉS : Optimisation multi-critère, Théorie des jeux, Optimisation concourante, Partage de Territoire, traitement d'image

KEYWORDS : Multi-criteria Optimization, Game theory, Concurrent Optimization, Split of territories, image processing

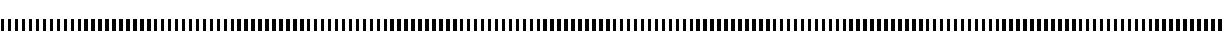




\section{Introduction}

On considére le problème d'optimisation multi-objective suivant :

$$
(P)\left\{\begin{array}{l}
\min _{y} f_{1}(y) \\
\min _{y} f_{2}(y)
\end{array}\right.
$$

où $f_{1}$ et $f_{2}$ sont deux fonctions convexes. L'idée est de partager l'ensemble des variables $y$ de minimisation en deux sous ensembles dont les variables seraient $U$ et $V$. Le but de ce partage serait de minimiser $f_{1}(U, V)$ par rapport à $U$ en fixant $V$ et de minimiser $f_{2}(U, V)$ par rapport à $V$ en fixant $U$, par une méthode d'optimisation concourante utilisant un algorithme simulant un jeu de Nash entre des joueurs associés aux deux fonctionnelles respectivement.On cherche alors à déterminer $y_{E N}=\left(U^{*}, V^{*}\right)$ solution du problème suivant

$$
(N)\left\{\begin{array}{l}
\min _{U} f_{1}\left(U ; V^{*}\right), \\
\min _{V} f_{2}\left(U^{*} ; V\right) .
\end{array}\right.
$$

Notre but est de trouver un équilibre de Nash sur le front de Pareto. La difficulté essentielle rencontrée par les algorithmes permettant de calculer un équilibre de Nash[3,9], est la détermination du meilleur partage de la variable $y$ en variables $U$ et $V$. Dans [4], J.A. Désidéri considère le cas où une des fonctionnelles est prépondérante, et propose un algorithme de partage de territoire original qui associe à la fonctionnelle secondaire des paramètres qui engendrent les plus grandes variations pour cette fonctionnelle tout en garantissant une moindre dégradation sur celle prioritaire.

\section{2. Équilibre de Nash}

Cette technique d'optimisation provient de la théorie des jeux et consiste à traiter les différents objectifs de manière concurrentielle comme des joueurs indépendants échangeant de l'information partielle. La recherche de l'équilibre de Nash se fait par échange des résultats obtenus pour chaque fonction objectif travaillant avec une partie seulement des variables, les autres étant fixées par les résultats obtenus pour les autres fonctions objectifs. Cet équilibre est atteint quand l'optimisation de chaque fonction conduit toujours à la même solution.

Dans ce qui suit on s'intéresse au cas d'un jeu à deux joueurs. Les deux joueurs cherchent à améliorer leurs propres critères parallèlement. Nous cherchons une stratégie $\left(U^{\star}, V^{\star}\right)$ qui définit l'état d'équilibre :

$$
\left\{\begin{array}{l}
f_{1}\left(U^{\star}, V^{\star}\right)=\min _{U} f_{1}\left(U, V^{\star}\right), \\
f_{2}\left(U^{\star}, V^{\star}\right)=\min _{V} f_{2}\left(U^{\star}, V\right) .
\end{array}\right.
$$


La stratégie $\left(U^{\star}, V^{\star}\right)$ est un équilibre de Nash.

Nous allons présenter deux algorithmes permettant de définir deux tableaux d'allocations $P$ et $Q$ pour définir les variables du premier joueur notées $U$ et les variables du deuxième joueur $V$.

\section{Algorithmes de partage}

Le vrai partage de la variable $y$ est de construire deux tableaux d'allocation $P$ et $Q$ de $\{0,1\}^{n}$ avec $P_{i}+Q_{i}=1$ pour $1 \leq i \leq n[1]$. Soit $I_{12}=\{1, \ldots, n\}$ un ensemble d'indices de cardinale $n, I_{1}$ un sous ensemble de $I_{12}$ de cardinale $n-p$, et $I_{2}$ son complémentaire de cardinale $p$, c'est-à-dire $I_{12}=I_{1} \cup I_{2}$.

Supposons que :

$$
\begin{cases}U=\left(y_{i}\right), & \text { pour } i \in I_{1}, \\ V=\left(y_{i}\right), & \text { pour } i \in I_{2} .\end{cases}
$$

On pose dans ce cas le tableau d'entiers d'allocation $P$ de taille $n$ :

$$
P_{i}=1, \forall i \in I_{1}, P_{i}=0, \forall i \in I_{2}
$$

de sorte que

$$
y=P . y+(\mathcal{I}-P) \cdot y=(U, V) \quad \text { avec } \mathcal{I}=(1, \ldots, 1) .
$$

Désignons par"." le produit d'Hadamard, c'est-à-dire $(P . y)_{i}=P_{i} y_{i}$ et $P . y \in \mathbb{R}^{n}$. Le vecteur $(U, V)$ est défini dans l'équation (3.4).

Le jeu de Nash $(\mathrm{N})$ peut s'écrire sous la forme suivante :

$$
(N)\left\{\begin{array}{l}
\text { Trouver } y_{E N} \text { solution de }: \\
\min _{U} f_{1}\left(P . y+(\mathcal{I}-P) \cdot y_{E N}\right), \\
\min _{V} f_{2}\left((\mathcal{I}-P) \cdot y+P . y_{E N}\right),
\end{array}\right.
$$

On considère le problème $\left(N^{\prime}\right)$ suivant :

$$
\left(N^{\prime}\right)\left\{\begin{array}{l}
\text { Trouver } y_{E N} \text { solution de : } \\
\min _{y} f_{1}\left(P . y+(\mathcal{I}-P) \cdot y_{E N}\right)+f_{2}\left((\mathcal{I}-P) \cdot y+P . y_{E N}\right),
\end{array}\right.
$$

$y_{E N}$ est un équilibre de Nash s'il est point fixe de $\left(N^{\prime}\right)[1]$.

Pour chaque choix de $P$ on trouve un équilibre de Nash, dans ce cas on a au plus $2^{n}$ équilibres de Nash. La question qui se pose est de savoir comment choisir parmi tous ces équilibres, un équilibre appartenant au front de Pareto s'il existe, ce qui n'est pas toujours le cas en général.

Dans ce travail, nous supposons que les éléments de $P$ appartiennent à $[0,1]$ et nous 
construisons un autre tableau $Q$ qui va remplacer le tableau $(\mathcal{I}-P)$ de telle sorte que $P+Q$ ne soit pas nécessairement égal à $\mathcal{I}$. Nous avons proposé et expérimenté avec succès deux algorithmex de partage (AG1) et (AG2) qui calculent les deux tableaux $P, Q$ et les équilibres de Nash associés. Dans l'algorithme de partage (AG1) nous avons partagé la variable d'optimisation $y$, en construisant deux suites de deux tableaux d'allocation $P^{(m)}$ et $Q^{(m)}$ dans $[0,1]^{n}$, les deux tableaux sont construits à partir de la minimisation itérative de $f_{1}$ et $f_{2}$, et de la minimisation de la fonction $f$ définie par l'équation (3.11). Dans l'algorithme de partage (AG2), les deux tableaux d'allocation $P$ et $Q$ sont considérés comme des variables d'optimisation. Il s'agit de présenter une formulation de jeux de Nash entre deux joueurs en utilisant les deux tableaux d'allocation $P$ et $Q$, et la variable $y$.

Dans ce qui suit, nous présentons les deux algorithmes proposés (AG1) et (AG2).

\subsection{Algorithme 1 (AG1) : Tableaux d'allocations heuristiques}

Dans ce qui suit, nous utilisons une approche heuristique pour construire les tableaux d'allocation. Elle est basée sur l'observation des directions de descente privilégiées par un algorithme de type gradient pour optimiser chacune des fonctionnelles séparément. Par exemple, la composante $P_{j}$ est la ratio du nombre de fois (relativement au nombre total d'itérations d'optimisation) où la direction $j$ a été utilisée pour diminuer le critère $f_{1}$.

Etape 1 :

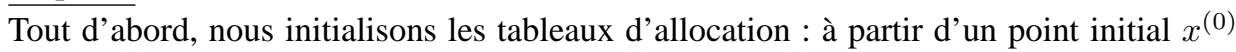
(resp. $\left.y^{(0)}\right)$ quelconque de $\mathbb{R}^{n}$, on calcule $P^{(0)}$ (resp. $Q^{(0)}$ ) par :

$$
\left\{\begin{array}{r}
\min _{x \in R^{n}} f_{1}(x), \quad x^{(k+1)}=x^{(k)}-\rho_{k} \nabla f_{1}\left(x^{(k)}\right), \quad k \geq 0, \\
P_{j}^{(0)}=\frac{\sum_{k}\left|x_{j}^{(k+1)}-x_{j}^{(k)}\right|}{\sum_{k}\left\|x^{(k+1)}-x^{(k)}\right\|}, \\
\min _{y \in R^{n}} f_{2}(y), \quad k \geq 0, \\
y_{j}^{(k+1)}=y^{(k)}-\rho_{k} \nabla f_{2}\left(y^{(k)}\right), \quad k y_{j}^{(k+1)}-y_{j}^{(k)} \mid \\
\sum_{k}\left\|y^{(k+1)}-y^{(k)}\right\|
\end{array}\right.
$$

où $\rho_{k}$ est un pas de descente.

Notons,

$$
y_{E N}^{(m)}=P^{(m)} \cdot x^{*}+Q^{(m)} \cdot y^{*}
$$

où,

$$
\left\{\begin{array}{l}
x^{*}=\operatorname{Arg} \min _{x} f_{1}(x) \\
y^{*}=\operatorname{Arg} \min _{y} f_{2}(y) .
\end{array}\right.
$$


On définit la fonction $f$ par

$$
f(x)=f_{1}(\bar{x})+f_{2}(\bar{y})
$$

où,

$$
\bar{x}=P^{(m)} \cdot x+Q^{(m)} \cdot y_{E N}^{(m)} \quad \text { et } \quad \bar{y}=P^{(m)} \cdot y_{E N}^{(m)}+Q^{(m)} \cdot x
$$

Etape 2 :

On construit $P^{(m+1)}$ et $Q^{(m+1)}$ tels que

$$
\left\{\begin{array}{c}
\min _{x} f(x), \quad \begin{array}{l}
(k+1)=x^{(k)}-\rho_{k} \nabla f\left(x^{(k)}\right) \quad k \geq 0 \\
P_{j}^{(m+1)}= \\
\sum_{k}\left|\left(\nabla f_{1}\left(P^{(m)} \cdot x^{(k+1)}+Q^{(m)} \cdot y_{E N}^{(m)}\right)\right)_{j}\right| \\
\sum_{k}\left\|\nabla f_{1}\left(P^{(m)} \cdot x^{(k+1)}+Q^{(m)} \cdot y_{E N}^{(m)}\right)\right\|
\end{array} \\
Q_{j}^{(m+1)}=\frac{\sum_{k}\left|\left(\nabla f_{2}\left(Q^{(m)} \cdot x^{(k+1)}+P^{(m)} \cdot y_{E N}^{(m)}\right)\right)_{j}\right|}{\sum_{k}\left\|\nabla f_{2}\left(Q^{(m)} \cdot x^{(k+1)}+P^{(m)} \cdot y_{E N}^{(m)}\right)\right\|} . \\
y_{E N}^{(m+1)}=P^{(m)} \cdot x_{o p t}^{(m)}+Q^{(m)} \cdot y_{E N}^{(m)}
\end{array}\right.
$$

où $x_{o p t}^{(m)}$ est la solution du $\min _{x} f(x)$.

Tant que $\left\|y_{E N}^{(m+1)}-y_{E N}^{(m)}\right\|>$ test, poser $m=m+1$, et répéter (3.12).

\subsection{Algorithme 2 (AG2) : Tableaux d'allocations optimaux}

Dans cette variante, les tableaux d'allocation ne sont pas remplis de façon heuristique, mais sont optimisés vis à vis du critère hybride $f$. Comme les valeurs optimisées de $P$ et $Q$ ne sont pas binaires, l'interprétation du résultat obtenu est délicate, et nous désignons encore par équilibre de Nash un point fixe de $f$, bien que ce ne le soit plus au sens habituel.

1. construction de $P^{(0)}$ et $Q^{(0)}$ en utilisant l'étape 1 de (AG1)

2. pour $P^{(m)}$ et $Q^{(m)}$ donnés, minimiser $f(x)$ par rapport à $x$, on obtient $x_{o p t}^{(m)}$

3. minimiser la fonction $f(x)$ par rapport à $P$, on obtient $P^{(m+1)}$

4. minimiser la fonction $f(x)$ par rapport à $Q$, on obtient $Q^{(m+1)}$

5. $y_{E N}^{(m+1)}=P^{(m+1)} \cdot x_{o p t}^{(m)}+Q^{(m+1)} \cdot y_{E N}^{(m)}$

6. tant que $\left\|y_{E N}^{(m+1)}-y_{E N}^{(m)}\right\|>$ test.

7. poser, $m=m+1$, retour à 2 . 
Notre but à présent est de montrer que le meilleur partage qu'on obtient par les deux algorithmes (AG1) et (AG2), donne un équilibre de Nash qui appartient au front de Pareto ou bien proche du front.

Le front de Pareto est l'ensemble des solutions non dominées[1]. Lorsque le front de Pareto est convexe, on peut trouver tous ses points en minimisant

$$
f_{w}=w f_{1}+(1-w) f_{2}, \quad w \in[0,1] .
$$

Pour tout $w$, on trouve un point d'équilibre au sens de Pareto.

Dans la partie suivante nous présentons quelques résultats numériques dans le cas ou $f_{1}$ et $f_{2}$ sont convexe, et pour des matrices symétriques définies positives en appliquant (AG1) et (AG2). Une autre application en traitement d'images en appliquant (AG1).

\section{Résultats Numériques}

On considère deux fonctions convexes de la forme suivante :

$$
f_{1}(x)=\|A x-b\|^{2} \text { et } f_{2}(x)=\|C x-d\|^{2}, x \in R^{n \times 1}
$$

où $A$ et $C$ sont deux matrices de taille $(n, n), b$ et $d$ deux vecteurs de taille $(n, 1)$, et $\|$. $\|$ est la norme euclidienne : $\|x\|=\left(\sum_{i=1}^{n} x_{i}^{2}\right)^{\frac{1}{2}}$

Dans l'étape 2 de l'algorithme (AG1), pour calculer le gradient de la fonction $f$ nous utilisons les technique suivantes :

$$
\bar{x}=P^{(0)} \cdot x+Q^{(0)} \cdot y_{E N}^{(0)} \quad \text { et } \quad \bar{y}=P^{(0)} \cdot y_{E N}^{(0)}+Q^{(0)} \cdot x .
$$

A l'itération $m$ de l'étape 2 , la fonction $f$ s'écrit sous la forme suivante :

$$
f(x)=\left\|A\left(P^{(m)} \cdot x+Q^{(m)} \cdot y_{E N}^{(m)}\right)-b\right\|^{2}+\left\|C\left(Q^{(m)} \cdot x+P^{(m)} \cdot y_{E N}^{(m)}\right)-d\right\|^{2},
$$

avec,

$P_{m}=\left(\begin{array}{ccccc}P_{1}^{(m)} & 0 & 0 & \ldots & 0 \\ 0 & P_{2}^{(m)} & 0 & \ddots & \vdots \\ 0 & 0 & \ddots & \ddots & 0 \\ \vdots & \ddots & \ddots & \ddots & 0 \\ 0 & \ldots & 0 & 0 & P_{n}^{(m)}\end{array}\right)$ et $Q_{m}=\left(\begin{array}{ccccc}Q_{1}^{(m)} & 0 & 0 & \ldots & 0 \\ 0 & Q_{2}^{(m)} & 0 & \ddots & \vdots \\ 0 & 0 & \ddots & \ddots & 0 \\ \vdots & \ddots & \ddots & \ddots & 0 \\ 0 & \ldots & 0 & 0 & Q_{n}^{(m)}\end{array}\right)$.

donc

$\nabla_{x} f(x)=2\left(A P_{m}\right)^{T}\left(A\left(P^{(m)} \cdot x+Q^{(m)} \cdot y_{E N}^{(m)}\right)-b\right)+2\left(C Q_{m}\right)^{T}\left(C\left(Q^{(m)} \cdot x+P^{(m)} \cdot y_{E N}^{(m)}\right)-d\right)$, 


\subsection{Test 1}

Dans ce test, on utilise les données suivantes :

$$
A=C=I d, b=[1 ;-2 ; 2 ; 9 ; 1 ; 2 ; 9], d=[5 ; 1 ; 3 ;-8 ;-6 ; 0 ; 4] .
$$
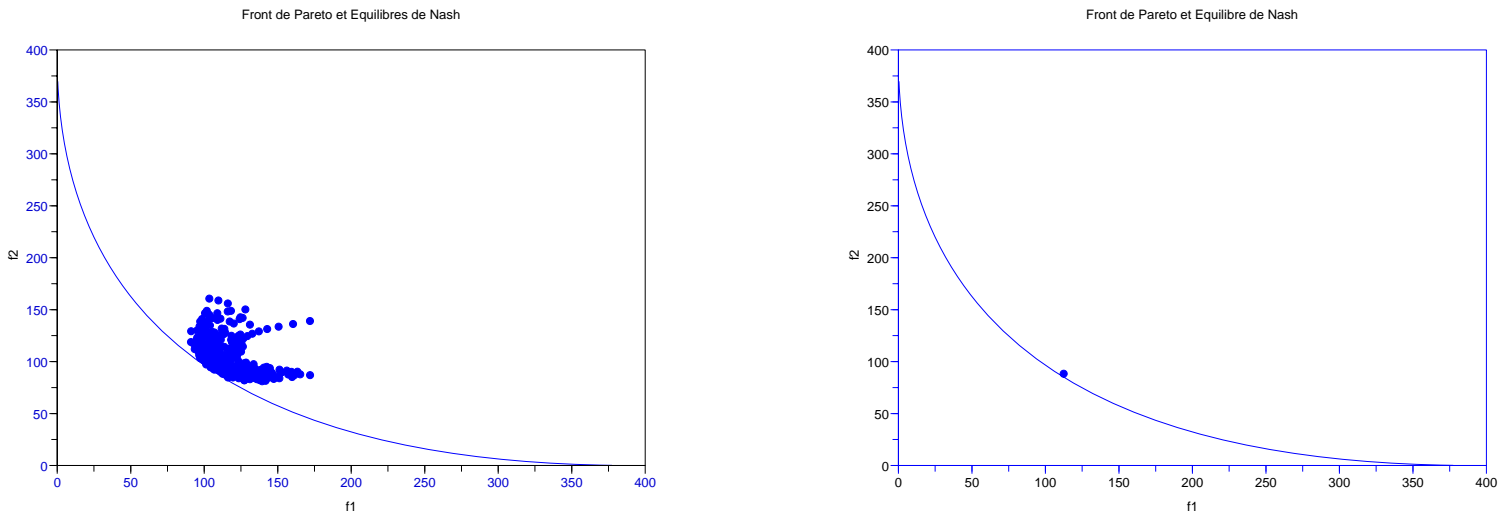

Figure 1 - A gauche, Front de Pareto et équilibres de Nash, A droite, dernier équilibre de Nash trouvé par (AG1)

\subsection{Test 2}

Dans ce test, on utilise les données suivantes :

$$
\mathbf{A}=\operatorname{tridiag}[1,-2,1], C=A ; b=\operatorname{rand}(n, 1), d=10 b, n=50
$$

On remarque que le dernier équilibre de Nash trouvé par (AG1) et (AG2) appartient au front de Pareto. 


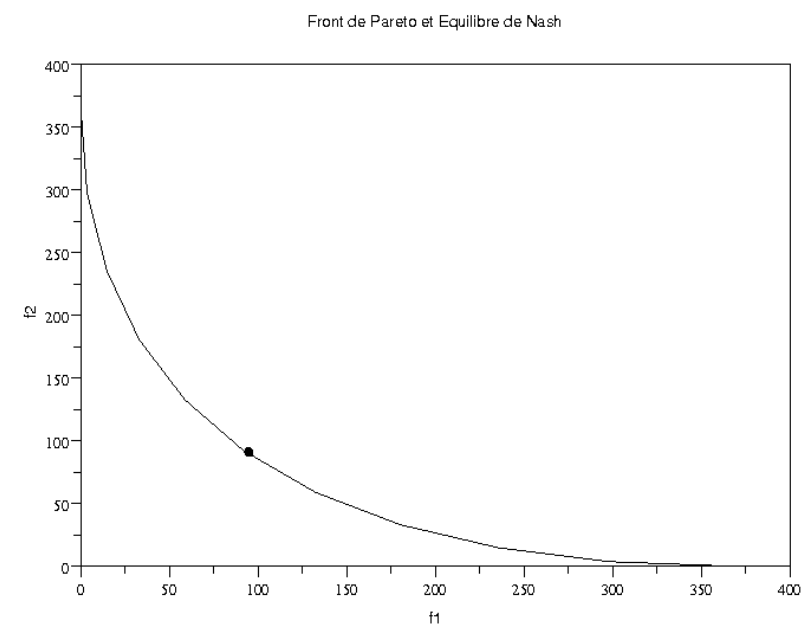

Figure 2 - Dernier équilibre de Nash trouvé par (AG2)

\subsection{Application au traitement d'images}

Soit $\Omega$ un ouvert borné, $y$ une fonction de $H_{0}^{1}(\Omega)$ et $F$ une image bruitée supposée dans $L^{2}(\Omega)$. A partir de l'image observée $F$ on cherche à reconstruire $y$ comme solution du problème de minimisation de :

$$
P(y)=\frac{1}{2} \int_{\Omega}|y-F|^{2} d x .
$$

Il s'agit d'un problème inverse mal posé. Pour le résoudre numériquement, on est amené à introduire un terme de régularisation, et à considérer le problème de minimisation de :

$$
P_{\varepsilon}(y)=\underbrace{\frac{1}{2} \int_{\Omega}|y-F|^{2} d x}_{\text {Ajustement aux données }}+\underbrace{\frac{\varepsilon}{2} \int_{\Omega}|\nabla y|^{2} d x}_{\text {Régularisation }} \text { (Tikhonov) }
$$

où $\varepsilon$ est un petit paramètre positif.

La minimisation de la fonctionnelle $P_{\varepsilon}$ passe par une diminution du gradient $\int_{\Omega}|\nabla y|^{2} d x$ donc un lissage global de la solution[4,10]. Les discontinuités sont de ce fait excessivement pénalisées et l'image estimée a un aspect trop lisse[4,10].

Le coefficient $\varepsilon$ permet de jouer sur le poids du terme régularisant par rapport au terme d'ajustement aux données, c'est un paramètre qui, pour une certaine valeur, assure une meilleure restauration de l'image[7,9]. Le choix de ce paramètre est important pour la qualité des résultats. Pour éviter ce choix, nous proposons une autre technique de régula- 

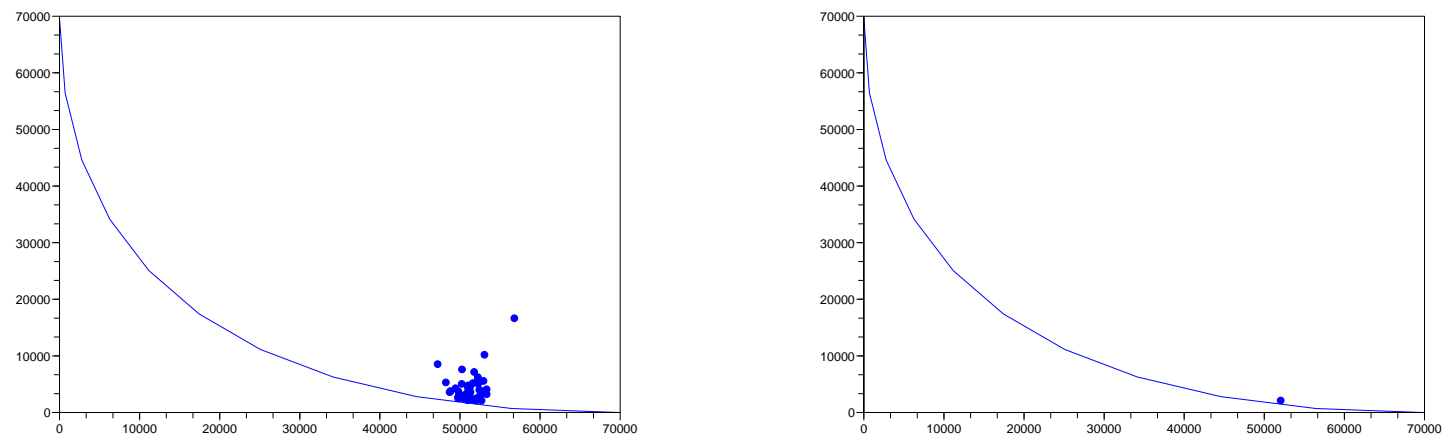

Figure 3 - A gauche, Front de Pareto et équilibres de Nash. A droite, dernier équilibre de Nash trouvé par (AG1)

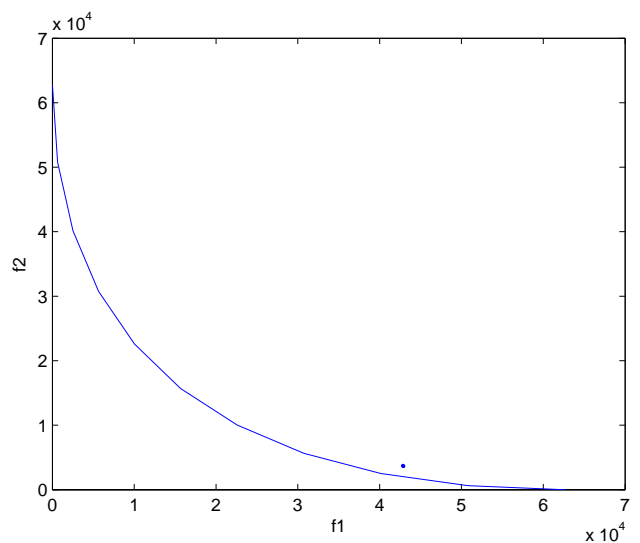

Figure 4 - Dernier équilibre de Nash trouvé par (AG2) 
Tableau 1 - L'importance du partage de la variable d'optimisation

\begin{tabular}{|c|c|c|c|c|c|}
\hline & Tikhonov & \multicolumn{4}{|c|}{ AG1 } \\
\hline SNR & 17.7189 & 17.5123 & 17.7234 & 16.8802 & 16.6415 \\
\hline Paramètres & $\varepsilon=0.1$ & $\lambda=0.1$ & $\lambda=0.01$ & $\lambda=0.001$ & $\lambda=0.0001$ \\
\hline
\end{tabular}

risation utilisant l'optimisation concourante basée sur le partage de territoire entre deux joueurs où le critère d'identification $f_{1}$ joue contre le critère de régularisation $f_{2}$ tel que :

$$
f_{1}(y)=\frac{1}{2}\|y-F\|^{2}+\frac{\lambda}{2}\|\nabla y\|^{2} \quad \text { et } \quad f_{2}(y)=\frac{1}{2}\|\nabla y\|^{2} \quad \forall y \in H_{0}^{1}(\Omega)
$$

où $\lambda$ est un petit paramètre fixé dans l'intervalle $[0,1]$, qui joue ici le rôle d'un paramètre de régularisation.

Parmi les critères quantitatifs les plus courants pour évaluer les performances des modèles de débruitage, nous utilisons le rapport signal-bruit (SNR) [5], exprimé en décibels et reliant l'image de référence $I_{1}$ et l'image $I_{2}$ après analyse par la relation :

$$
\operatorname{SNR}\left(I_{1} / I_{2}\right)=10 \log _{10}\left[\frac{\sigma^{2}\left(I_{1}\right)}{\sigma^{2}\left(I_{1}-I_{2}\right)}\right] .
$$

Pour obtenir la meilleure solution du problème $P_{\varepsilon}$ nous varions le paramètre $\varepsilon$ et calculons le rapport signal-bruit (SNR). La figure 5 présente l'évolution du SNR en fonction de $\varepsilon$, pour $\varepsilon=10^{-i+1}, i=1: 10$. Nous remarquons que la meilleure valeur, $\varepsilon=0.10$, correspond à une valeur du $S N R=17.7189$. La courbe présentée montre que des variations faibles autour de la valeur optimale de $\varepsilon$ peuvent dégrader sensiblement le SNR.

Les résultats obtenus en utilisant l'algorithme AG1 sont présentés dans le tableau 1, pour différentes valeurs de $\lambda$. Pour la résolution du problème de traitement d'images considéré, nous utilisons l'algorithme AG1, sachant que les deux algorithmes AG1 et AG2 ont donné des résultats simillaires pour les exemples considérés. 


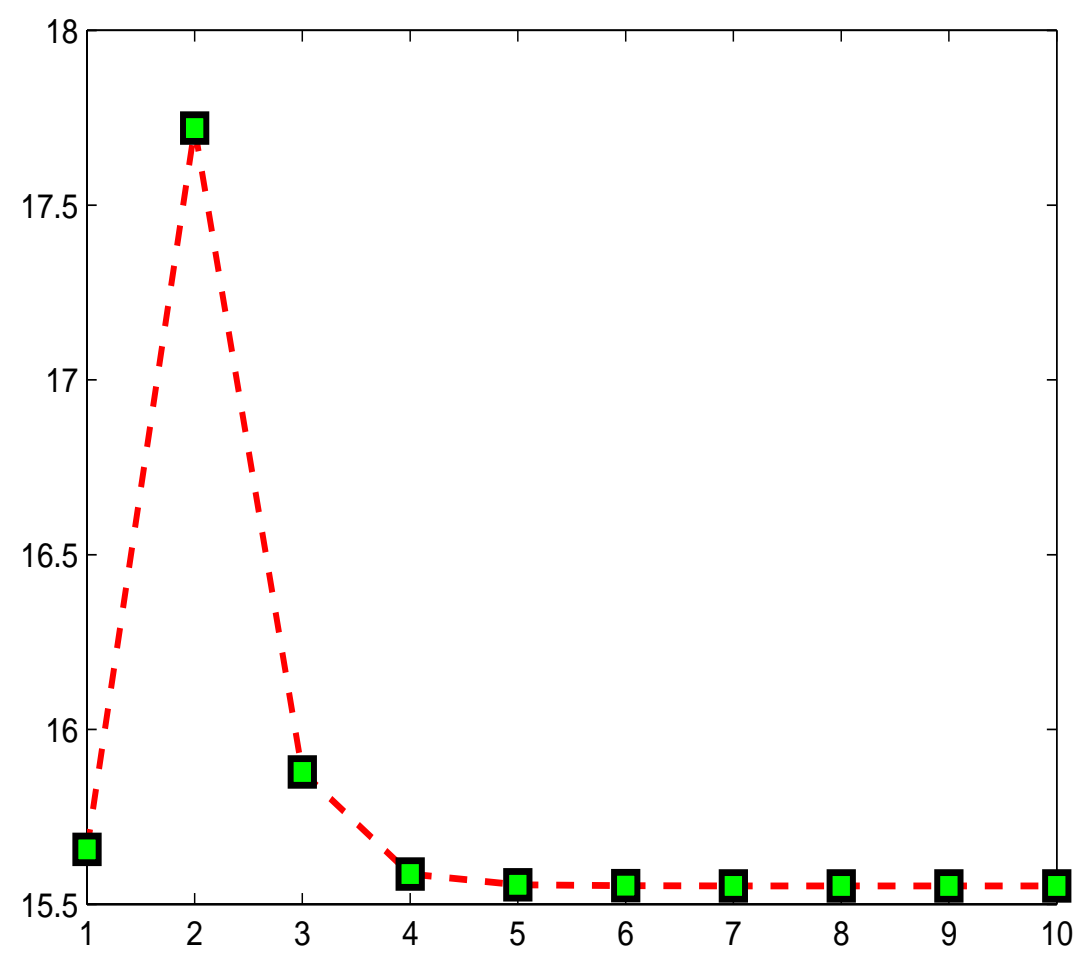

Figure 5 - L'évolution de la SNR en fonction de l'index de test $i=1: 10$ avec $\varepsilon=10^{-i+1}$

On remarque que l'algorithme (AG1) donne des résultats robustes par rapport aux variations de $\lambda$ contrairement à la régularisation par Tikhonov.

\section{Conclusion}

Dans ce travail nous avons présenté deux algorithmes de partage de variable d'optimisation, le premier construit deux tableaux d'allocations heuristiques et l'autre construit deux tableaux d'allocations optimaux. Après la convergence, on obtient un équilibre de Nash appartenant au front de Pareto dans le cas des exemples considérés. Les algorithmes proposés permettent d'affecter à chaque fonction $f_{i}, i=1: 2$ sa variable d'état à l'aide des tableaux d'allocation $P$ et $Q$ construits automatiquement. Les premiers résultats obtenus par l'algorithme (AG1), pour le traitement d'image sont encourageants. 


\section{Remerciements}

Ce travail a été supporté par l'Action Intégrée Franco-Morocaine MA/05,115, le Programme SARIMA et le projet $\mathrm{SCO} \mu \mid \mathrm{COADVISE}$.

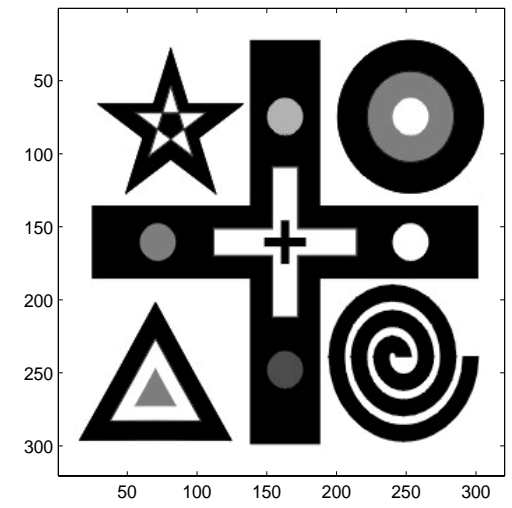

(a) Image originale

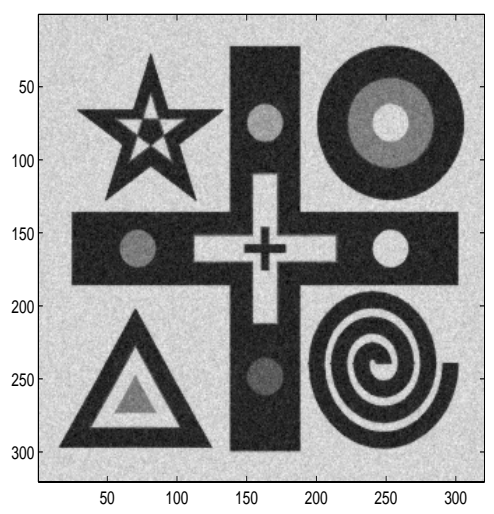

(c) Image restaurée par Tikhonov

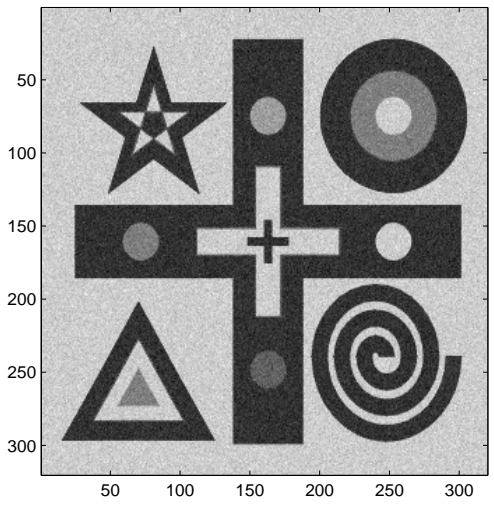

(b) Image bruitée

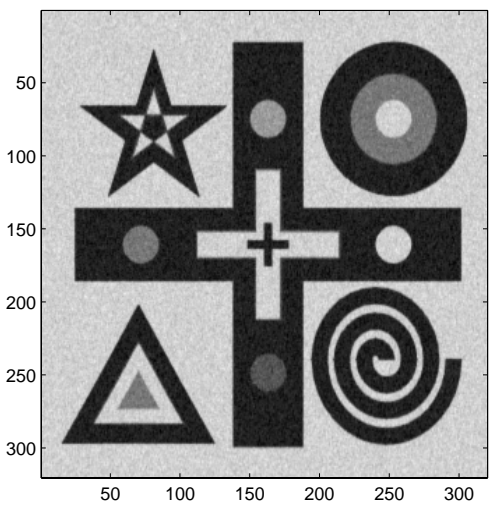

(d) Image restaurée par (AG1) $(\lambda=0.1)$ 


\section{Bibliographie}

[1] Aboulaich R., Habbal A., Moussaid N., Split of an optimization variable in game theory, Math. Model. Nat. Phenom(MMNP), accepted

[2] Allaire G. Conception optimale de structures, Mathématiques et Applications, Springer Verlag, Heidelberg,2006

[3] Aubin J.P., Mathematical methods of game and economic theory. North-Holland Publishing Co. Amsterdam, New York, 1979.

[4] Désidéri J.A., Split of Territories in Concurrent Optimization. Rapport de recherche, Institut National de Recherche en Informatique et en Automatique , N ${ }^{\circ}$ 6108, 8th October 2007.

[5] Destuynder Philippe, Cours d'Analyse, Traitement et Synthèse d'Images Numériques. Calcul Scientifique CNAM Paris 292 rue saint Martin 75003.20 mars 2005

[6] El Guarmah E., Sellami H., A comparison between a linear approach and a nonlinear diffusion model in image restoration. IJAM vol. 22, $\mathrm{n}^{\circ} 1,2009$

[7] A. Habbal, J. Petersson and M. Thellner, Multidisciplinary topology optimization solved as a Nash game. Int, J. Numer. Meth. Engng 2004 ; 61 :949-963

[8] P. Destuynder, M. Jaoua, and H. Sellami, A dual algorithm for denoising and preserving edges in image processing. J. Inv. Ill-Posed Problems 15 (2007), 19 - 35.

[9] A. Habbal, H. Lievre, J.A Desideri, Optimisation numérique de profils d'aile par algorithmes genetiques et jeux de Nash. Reserach report number 4275, INRIA, septembr 2001.

[10] Jean-François Aujol, Contribution à l'analyse textures en traitement d'images par méthodes variationnelles et équations aux dérivées partielles.. Thèse, Université de Nice-Sophia Antipolis, 2004.

[11] Tikhonov, A.N., Goncharsky, A.V., Stepanov, V.V., Yagola, A.G., Numerical methods for the solution of ill-posed problems, . Kluwer Academic Publishers, 1995. 